\title{
INTEGRATING ERRORS INTO THE TRAINING PROCESS: THE FUNCTION OF ERROR MANAGEMENT INSTRUCTIONS AND THE ROLE OF GOAL ORIENTATION
}

\author{
DOERTE HEIMBECK, MICHAEL FRESE, SABINE SONNENTAG \\ Department of Psychology \\ University of Amsterdam, Netherlands \\ NINA KEITH \\ Department of Psychology \\ University of Giessen, Germany
}

\begin{abstract}
Error management training explicitly allows participants to make errors. We examined the effects of error management instructions ("rules of thumb" designed to reduce the negative emotional effects of errors), goal orientation (learning goal, prove goal, and avoidance goal orientations) and attribute $\times$ treatment interactions on performance. A randomized experiment with 87 participants consisting of 3 training procedures for learning to work with a computer program was conducted: (a) error training with error management instructions, (b) error training without error management instructions; and (c) a group that was prevented from making errors. Results showed that shortand medium-term performance (near and far transfer) was superior for participants of the error training that included error management instructions, compared with the two other training conditions. Thus, error management instructions were crucial for the high performance effects of error training. Prove and avoidance goal orientation interacted with training conditions.
\end{abstract}

Errors appear in nearly every learning process because of insufficient knowledge and skills. Moreover, errors appear not just as a result of too little knowledge but also as a consequence of inappropriate goals and plans, interruptions during the action process, and inaccurate interpretation of system feedback (Zapf, Brodbeck, Frese, Peters, \& Prümper, 1992). Errors are often frustrating and can lead to anger and despair (Brodbeck, Zapf, Prümper, \& Frese, 1993).

This study is based on the dissertation of the first author in partial fulfillment for the doctoral degree at the University of Amsterdam. Doerte Heimbeck has since moved to Florida, U.S., Michael Frese has moved to University of Giessen, Germany, and Sabine Sonnentag has moved to University of Braunschweig, Germany. We are indebted to Rick DeShon for a critical reading of a prior version of this article.

Correspondence and requests for reprints should be addressed to Michael Frese, University of Giessen, Germany Otto-Behaghel-Str. 10F, 35394 Giessen, Germany; Michael.Frese@Psychol.Uni-Giessen.de 
Because errors appear frequently in the learning process, one would imagine that there is a strong literature on errors and the function of errors in training. However, this is not the case. Only very recently has the function of errors in training been explicitly discussed (e.g., Gully, Payne, Koles, \& Whiteman, 2002; Ivancic \& Hesketh, 2000). Traditionally, there was little explicit discussion of errors in the training literature and the little discussion there was, was primarily negative. For example, Skinner (1953) equated errors with punishment and argued that punishment leads to emotional arousal and to temporary suppression of behavior but does not tell the learner what needs to be done. Therefore, Skinner (1953) argued for an error prevention approach that minimizes errors; eventually his approach led to the development of the famous programmed learning machines that were supposed to maximize positive reinforcement and shaping by positive reinforcement (Skinner, 1968). Critiques of exploratory learning (e.g., Ausubel, 1968) also warned of the dangers of errors in the learning process; they suggested (among others) that allowing people to make errors lets them practice incorrect and inefficient approaches; this was one reason that exploratory learning was deemed to be inefficient in comparison to teaching appropriate cognitive models (e.g., Ausubel, 1968; similarly also Debowski, Wood, \& Bandura, 2001). Discussions with software trainers and informal observations of their training processes have also convinced us that most software trainers attempt to prevent errors. Software trainers thought that errors would lead to negative effects and they studiously avoided them whenever possible. Thus, errors are often seen to be "nuisance" factors that need to be prevented in training and, at the same time, there are relatively few explicit studies of errors in training.

The approach taken in this article is to counter the error prevention approach. We conceptualize errors as as source of negative feedback and argue that errors can have a positive and informative function in training. Thus, errors should not be avoided but explicitly incorporated into the training process. The training concept suggested here is one of error training - a training method suggested by Frese and coauthors, which increases the exposure to errors but which also produces a safe environment for errors to appear (Frese, 1995; Frese \& Altmann, 1989; Frese et al., 1991). Error training proved to lead to higher performance than a training that reduced the chances to make errors, the so-called error-avoidant training (e.g., Frese et al., 1991). Recent research has been a bit more skeptical about error training (Debowski et al., 2001; Gully et al., 2002). Debowski et al. (2001) showed that enactive exploration (an approach similar to error training) produced weaker performance effects than what they called guided exploration. However, with more extensive training time, the performance results of both training 
procedures may become similar (Wood, Kakebeeke, Debowski, \& Frese, 2000). Debowski et al. (2001) also discussed self-regulatory and affective processes and suggested that such processes should be studied more frequently in training research. Gully et al. (2002) did not find a general performance superiority of error training but rather attribute-treatment interactions; positive performance effects of error training (as compared to an error avoidance training) occurred for high ability and high openness to experience people. Gully et al. (2002), therefore, argue to include interindividual difference variables into training design research.

The current study takes the error training approach as a starting point and advances our knowledge in four areas: First, it replicates the studies done so far; this replication is of special interest because some other studies have revealed a somewhat more ambiguous picture about the effectiveness of error training (Debowski et al., 2001). Second, the study explicitly examines the function of the error management instructions that are supposed to reduce the negative emotional effects of errors. Third, the study examines the effects of error training on two transfer outcomes, including a far transfer outcome obtained one week following the conclusion of the training. Fourth, we examine goal orientation as a specific attribute in an attribute-treatment interaction.

\section{Error Training: Conceptual and Empirical Issues}

The error training concept starts from the assumption that errorsdefined as deviations from goals that are potentially avoidable-provide feedback and that negative feedback is a necessary prerequisite for learning (Frese \& Zapf, 1994). Feedback as a learning device is also the basis of exploratory learning (Bruner, 1966; Carroll, Mack, Lewis, Grischkowsky, \& Robertson, 1985; Greif \& Janikowski, 1987; Greif \& Keller, 1990). Exploratory learning is similar to error training, although error training tends to present more difficult tasks earlier in training. Although most error training programs also increase difficulty level during the course of the training, both the starting and the endpoint tasks are usually more difficult in error training than within the exploratory learning paradigm. A first evaluation of error training of a software system was provided by Frese et al. (1991); in this study, the error training group had ample opportunities for making errors by having to solve tasks that were too difficult for the participants. Error training provides trainees with only the most basic information and minimal instructions about the program taught during the training. The error training group in Frese et al.'s study received only a list of commands that could be used to deal with the tasks. Therefore, this group had to try out several approaches and to explore the system until they found a way to solve the tasks. Dur- 
ing this process, participants made a number of errors. We call them errors because participants could not reach the goal of solving the tasks and knew that there was, in principle, a solution available (cf. Frese \& Zapf, 1994, for a concept of errors). Examples of errors are that participants attempted to use the wrong commands (e.g., CTRL T instead of CTRL D to delete a letter) or that they used a keystroke combination that led the participants into unknown parts of the software (e.g., getting into the area of commands for margin setting instead of changing letters in a text). In contrast, the error avoidant group was given detailed instructions of how to solve the tasks. Because these detailed instructions were correct, they did not make any errors; if an error occurred in spite of these detailed prescriptions of actions (e.g., because of a typing error or wrong reading of the instructions), the trainer intervened and corrected the situation immediately-making sure that the participants had minimal exposure to an error situation. After the training, a performance test was done that examined whether participants could solve everyday tasks of moderate to high difficulty. For example, in the word processing training, participants were tested to set margins or design a text (Frese et al., 1991). The error training group showed higher performance in tasks of medium and high difficulty than the group that received erroravoidant training (Dormann \& Frese, 1994; Frese et al., 1991; Greif \& Janikowski, 1987; Ivancic \& Hesketh, 2000). Performance in easy tasks did not differ between error and error avoidant trainings (Frese, 1995). Easy tasks require a low degree of skill and, therefore, do not normally lead to errors; thus, they are less affected by error training.

Some researchers have argued that error training would be particularly useful to increase transfer of training (Frese, 1995; Hesketh, 1997). Because successful transfer represents the most important training outcome (Baldwin \& Ford, 1988; Goldstein, 1992), an effective training method should produce high transfer. Transfer implies that there is a gap between learning tasks and transfer tasks (Gick \& Holyoak, 1987; Kraiger, 1995). Gick and McGarry (1992) found a positive relationship between failure during the source problem solving and transfer in an analogous problem-solving experiment. Most training research assumes that learning and transfer occur in different environments: The learning context is supposed to be safe and well structured. Tasks are carefully sequenced and described, and feedback is provided in such a way that skill acquisition is facilitated. In contrast, the transfer context is open, disruptive, and ambiguous (Kraiger, 1995). Thus, the transfer situation introduces the chance to make errors. Trainees have to deal with errors, explore new aspects of the program, and solve new and more difficult tasks in the transfer environment. In this environment, help is typically not provided, nor is there enough time to look through an extensive man- 
ual (Brodbeck et al., 1993). Error training reduces the distance between training and transfer environments as it allows and encourages errors to occur in the training process, teaching skills to deal with errors in the training context. Thus, error training should facilitate transfer performance.

\section{Error Management Instructions}

In some ways, error training is frustrating to its participants. Frese et al. (1991) presented in the 5th and 6th training hour tasks that had been pretested not to be achievable by any of the participants (for example, to write the Danish currency "Øre" with the system Wordstar of the 1980s). Participants usually attempted various approaches but none of them achieved the goal of writing "Øre." Participants were aware that, in principle, there was a solution. To reduce the frustrating role of this situation, error management instructions were used by explaining to the participants that errors were necessary to learn and presenting the following four error management instructions: "I have made an error. Great!"; "There is always a way to leave the error situation"; "Look at the screen"; "I watch what is on the screen and what is changing." These instructions were developed to counter ineffective emotions that appeared in pilot studies in which people's reactions to errors were observed. For example, people were sometimes deeply frustrated by errors and they had the feeling that they could not get out of certain "error traps." People also tended not to look at the screen and not to observe what had changed on the screen and thereby minimized the feedback they received from errors (Frese \& Altmann, 1989). The error management instructions were displayed prominently and were reiterated by the trainers throughout the training.

Why should error training with error management instructions lead to high performance? Our argument is twofold. First, we suggest that using errors in training helps to develop a good operative mental model. However, this positive effect can only appear if training participants are not hindered by the negative emotional effects of errors. Second, these negative effects can be countered by error management instructions. Thus, only the combination of error training and the error management instructions should lead to high performance.

First, the development of a good mental model is enhanced by error training. Errors provide negative feedback and point out areas of misunderstandings. Therefore, errors can lead people to develop a better mental model of the system (it is an operative mental model as the model is supposed to regulate behavior adequately; Frese \& Zapf, 1994). Errors interrupt the behavior stream and the interruption of the behavior 
stream helps the person to develop thoughtful strategies to learn and enhance the mental model (Lord \& Levy, 1994). The chance to make errors increases deeper level processing or elaborate rehearsal (Craig \& Lockhart, 1972), leading to better memory for the mental model developed. The interruption of the action stream may, in addition, lead to the use of deliberate practice. Deliberate practice is an effortful practice of issues that one has not yet mastered (Ericsson \& Charness, 1994; Ericsson, Krampe, \& Tesch-Römer, 1993). Thus, the training participants deliberately practice those areas in which they have made errors leading to better mental models. Further, error training allows people to learn and practice error recovery strategies (such as using the UNDO function of the computer), which increases performance. Errors may also lead to more exploration; when an error occurs, people tend to explore the system because people want to know why the error occurred or in which part of the system the error happened (Dormann \& Frese, 1994). Thus, all these factors explain why errors help in the development of a good mental model, which, in turn, helps to regulate effective actions.

Second, errors lead to stress and frustration and, therefore, the stressfulness of the errors need to be countered to make it possible to learn from errors. Modern stress theory suggests that information processing resources allocated to the task are overtaxed when negative emotions, worrying, ruminating, and so forth have to be dealt with because they represent additional tasks (Hockey, 1996; Meijman \& Mulder, 1998). Because the error management instructions reduce worrying and ruminating about errors, they should reduce the negative effects of errors and should make the person open to learn from error feedback, to explore, and to deliberately practice. Empirically, the frustration level of the error training participants (which included the error management instructions) was lower in the performance phase than the frustration level of the error avoidant participants in two studies (Frese et al., 1991; Nordstrom, Wendland, \& Williams, 1998).

The error management instructions can be understood within the Cannon-Bowers, Rhodenizer, Salas, and Bowers' (1998) framework on conditions before, during, and after practice. Within Cannon-Bowers et al.'s framework, error management instructions are an attentional device and produce metacognitive strategies to better self-regulate errors. An example that using metacognitive strategies can be beneficial for performance is provided by Ford, Smith, Weissbein, Gully, and Salas (1998). Similarly, the error management instructions should provide a metacognitive framework of how to think of errors and to conceptualize errors in a positive way. As a matter of fact, Ford et al.'s (1998, p. 224, 225) measure of metacognitive activity included items that we would interpret to measure a sort of error management orientation, for example: 
"I noticed where I made the most mistakes during practice and focused on improving those areas." Metacognitive activity was positively related to both training and transfer performance (Ford et al., 1998).

No study has yet examined the effects of the error management instructions and this article, therefore, tests the function of instructions explicitly. In our original work, the very concept of error training included error management instructions (Frese, 1995). Only the combination of allowing errors to appear and error management instructions should have a positive training effect, leading to the following hypothesis:

Hypothesis 1: Error training with error management instructions leads to higher performance than error training without instructions or error avoidant training.

\section{Error Training and Goal Orientation}

Recently, Gully et al. (2002) argued for testing attribute-treatment interactions in training research and showed that the effectiveness of error training depends upon personality and cognitive ability (cf. also the call by Tannenbaum \& Yukl, 1992, to examine attribute-treatment interactions). One potential motivational personality variable that may influence the effectiveness of error training is learning goal orientation (Cannon-Bowers et al., 1998). Dweck and coworkers (Dweck, 1986; Dweck \& Leggett, 1988) distinguished learning goals and performance goals in learning and achievement situations. Individuals with a learning goal orientation strive to increase their level of competence. They are interested in acquiring new skills and mastering new situations. People with high learning goal orientation take the occurrence of errors as a chance to learn (Dweck \& Leggett, 1988). In contrast, individuals adopting a performance goal orientation are more concerned with demonstrating good performance and gaining favorable judgment, and perceive errors as an indicator of poor performance (Dweck \& Leggett, 1988).

A growing body of research describes the relationships between goal orientation, motivation, and performance. For example, Boyle and Klimoski (1995) found goal orientation and early training experience to influence trainees' persistence, task interest, satisfaction with task performance, and skill acquisition: If people showed only high performance goal orientation, learning was impaired. When high performance and high learning goal orientation coexisted, trainees acquired more knowledge and skills (cf. also Ames \& Archer, 1988). Goal orientation was related to the type of goals participants in a training course developed (Brett \& VandeWalle, 1999). Differences in goal orientation significantly predicted the level of attributional self-evaluation (Brown \& 
Kozlowski, 1997). Colquitt and Simmering (1998) showed that learning goal orientation was positively related to motivation to learn, whereas performance goal orientation was negatively related to motivation to learn. Learning goal orientation also functioned as a moderator: There was a negative relationship between prefeedback learning level and postfeedback expectancy in low learning orientation and no relationship in high learning orientation. Learning goal orientation was associated with high persistence in the face of failure, which led to higher skill development (a reversed moderator effect occurred for performance orientation on the relationship between prefeedback learning level and postfeedback values; Colquitt \& Simmering, 1998). Thus, people with a high learning goal orientation were buffered from reducing their expectancy when difficulties arose (when they received negative feedback on the first course exam). In sum, participants' goal orientations influence performance via motivational and cognitive processes. Thus, goal orientation is an important attribute to be considered in training studies.

Recent empirical evidence suggests that goal orientation is best represented by three dimensions (Elliot \& Harackiewicz, 1996; Heimbeck, Frese, Sonnentag, Van Dyck, \& Van der Linden, 2003; VandeWalle, 1997). VandeWalle (1997) kept the dimension of learning goal orientation, but subdivided performance goal orientation into two dimensions: prove and avoidance goal orientation. Prove goal orientation covers a positive, proactive, and approaching attitude towards task performance. People who are high on prove goal orientation prefer situations in which they can "shine" and show how well they can perform. In contrast, avoidance goal orientation increases people's propensity to evade negative judgments of performance and disapproval (this is similar to the distinction between hope for success and fear of failure in achievement motivation research; McClelland, 1987). Thus, high avoidance goal orientation should make people feel good in a situation in which they cannot make errors or in which difficult problems carry a high failure rate.

We expect a moderator effect for goal orientation in the sense of an attribute-treatment interaction (Eysenck, 1996; Snow, 1986). Our argument follows the reasoning developed with regard to error management instructions (see above): Learning orientation should make it possible to learn well within an error training framework because it reduces the negative emotional effects of errors. Learning orientation should be positive in error training because learning goal orientation implies that new and difficult tasks are interpreted as challenging learning opportunities (Dweck, 1986, 1989; Farr, Hofmann, \& Ringenbach, 1993). Such a persistent, active, explorative, and learning-oriented behavior should be beneficial for participants in the two error training conditions when they are confronted with error situations. 
In contrast, participants in the error avoidant condition should profit from high prove and avoidant goal orientation. Individuals scoring highly on prove goal orientation want to be right and show high performance - this is quite adaptive in an error avoidant group. The error avoidant training allows people to be right; immediate high performance is possible because every step is clearly pointed out by the written instructions. Therefore, high prove goal orientation should lead to better performance in the error avoidant condition than low prove goal orientation. Similarly, people high in avoidance goal orientation should also do better in the error avoidant condition, because avoidance goal oriented individuals try to avoid low performance and negative feedback and new and difficult tasks are perceived as threatening. The error avoidant condition is not threatening (because all answers are correct), there is no negative feedback and the tasks are easier because a description of how to solve them is given. Thus, it provides the environment in which high avoidance goal oriented individuals can develop good performance.

Hypothesis 2: Goal orientation and training conditions interact to produce attribute-treatment interactions: High learning goal orientation should be beneficial for performance (and low learning goal orientation should be detrimental) in error training conditions, high prove and avoidance goal orientation should be beneficial (and low prove and avoidance goal orientation should be detrimental) in the error avoidant condition.

\section{Method}

\section{Participants}

A total of 87 undergraduates from several disciplines at a German university participated in the experiment. Psychology majors among them received study points for participation ( $73.6 \%$ of the sample). Students from other disciplines received 20 DM (approx. \$10). Eighty percent of the sample were women. The average age was 23.55 years $(S D=3.89)$ with a range from 19 to 37 years. Participants varied widely in their experience with computers and use of software packages. To equalize knowledge with regard to the specific program taught, we only allowed participants into the training program who had never before worked with the software package taught in our training.

\section{Training Design}

The software package taught was a spreadsheet program (Excel 7.0 for Windows) used on a standard personal computer. Every action per- 
formed on the computer screen was recorded in real time on video. Participants were trained individually and were randomly assigned to one of three training conditions described below ( $n=29$ in each group): The participants of the error training with error management instructions group received instructions at the beginning of the training that emphasized the positive role of errors during training, instructions to make errors and to learn from them, and the following error management instructions, which we derived from earlier error training studies (Dormann \& Frese, 1994; Frese et al., 1991): "Errors are a natural part of the learning process!", "They inform what you are still able to learn!", "There is always a way to leave an error situation!", and "The more errors you make, the more you learn!" These error management instructions were prominently displayed on a poster next to the computer screen and they were repeatedly presented during the course of the training.

Participants in the error training without instructions condition participated in the same type of training as the group described above, but they were not encouraged to make errors and to learn from them, and they did not receive the error management instructions. Error avoidant training minimized the possibilities to make errors. Detailed written instructions guided the participants step by step through the training tasks so that all training tasks could be solved without making errors. There were a few participants in this group who finished the training early because time-consuming errors did not occur as in the other two groups. We kept training time constant for all three groups by asking these participants to work through the material again; this happened only in the error avoidant group.

The experimenter did not provide any help. If participants asked for help, the experimenter behaved in accordance with the experimental condition. In the error training with error management instructions, the participants were told to think of their instructions and to use the errors as instruments to learn from-no further help was provided. In the error training without these instructions, the experimenter explained that she could not help. In the error avoidant condition, the written instructions made it possible to solve all training tasks without errors. However, in some cases, participants of the error avoidant group used the wrong key in spite of the detailed instructions. In those cases, the experimenter immediately intervened and corrected the error so that the person did not get a chance to learn from the error (from informal observations of computer trainings, we had found experienced computer trainers to use such an approach in the normal course of their training as well). No further explanation was given. 


\section{Procedure}

The experiment consisted of four parts: first, assessment of questionnaire measures a few days before the experiment; second, the training phase of 45 minutes; third, the first performance test right after the training (45 minutes); and fourth, a second (transfer) performance test a week after the training (45 minutes) and debriefing. In the first phase, participants filled out the questionnaire on goal orientation and experience with computers at home. In Phase 2, all participants worked on the same training tasks under different training conditions. All participants received three minimal manuals describing important principles and functions of the program to be taught (Carroll, Smith-Kerker, Ford, \& Mazur-Rimetz, 1987; Lazonder \& van der Meij, 1993). After reading the text, a set of tasks was presented. The training started with tasks of low complexity (e.g., entering data, moving and copying data) and ended with more complex tasks (e.g., formatting tables and doing simple calculations). This phase lasted 45 minutes for all participants. In the third phase, the first performance test was done with a new set of performance tasks to be completed within 45 minutes. These tasks were designed to measure near transfer. They were similar to the tasks performed during the training, however they were more complex (e.g., complex data entry, rounding currencies, calculations, and producing the first diagram). During this test phase, none of the participants had any access to the manuals, to the instructions, or to the help of the experimenter. The fourth phase of the experiment took place approximately one week after the training. Here the participants worked on far transfer performance tasks for 45 minutes. These tasks were more difficult than those in the first test phase (e.g., doing calculations with complex if-then relationships, sorting data according to different criteria, copying data to additional sheets, complex formatting of a table, complex diagram with only partial information in the table). Before the participants left the lab, they received money or study points and were debriefed about the purpose of the experiment.

The training and performance tasks were the same for all. The training tasks consisted of starting Excel, entering data into an Excel table and saving it, correcting a table that was wrongly aligned, copying data from one part of the table to another part, and formatting (highlighting and underlining). Participants also had to add an additional column and had to calculate the sales of individuals. The performance test directly after the training consisted of producing a table, the calculation of the complete profit for six products, the calculation of the price of each piece within a product line, the calculation of the average profit rate for the three most expensive products, rounding numbers with the 
help of the Excel assistant, producing numbers with commas and points (e.g., \$13,495.45), and, finally, producing a circle diagram (again with the help of the Excel assistant). The transfer test one week after the training consisted of seven tasks, for example, to calculate the sales of each department, to calculate a wage increase of $100.00 \mathrm{DM}(\$ 50.00)$ for each person who had a certain sales volume, to produce a table that listed employees by age, and to reformat the table. The tasks were independent of each other so that one could fail to perform one and still do the next one.

\section{Measures}

Goal orientation. Goal orientation was measured with the 13-item work domain trait goal orientation instrument by VandeWalle (1997) that differentiates three dimensions: learning goal orientation, prove goal orientation, and avoidance goal orientation. Because nearly all German students have (repeated or continuous) work experience and because they use the term "working" for doing their studies, we decided to use the work domain goal orientation instrument. Sample items were: for learning goal orientation "I enjoy challenging and difficult tasks at work where I'll learn new skills"; for prove goal orientation "I try to figure out what it takes to prove my ability to others at work"; and for avoidance goal orientation "I prefer to avoid situations at work where I might perform poorly." Item responses were all based on a 5-point Likert scale ranging from $1=$ strongly disagree to $5=$ strongly agree. The goal orientation instrument was translated from English into German, retranslated into English and, based on this retranslated version, the German version was again improved by the authors and their research colleagues. Cronbach's alphas were .85 for learning goal orientation ( 5 items), .62 for prove goal orientation (4 items), and .76 for avoidance goal orientation (4 items).

Performance. Performance ratings were done on the basis of videotapes categorizing small units of task completion, which were single actions that could be observed and rated. For example, the task to create a certain table in Excel was divided into the following units: opening Excel, entering data correctly, and formatting the table. Every single unit was rated as either correctly completed or not (dichotomous rating item). Performance ratings were made by the experimenter and a second rater who was not familiar with the research question. For all single units during the training, average Cohen's Kappa was $.75(S D=.29)$, during the first performance test average Cohen's Kappa was $.82(S D=.21)$, and during the second test average Cohen's Kappa was .80 $(S D=.16)$. This produced an overall average Kappa for all three phases of .79 
$(S D=.22)$. These are good to excellent interrater agreements (Landis \& Koch, 1977). Further, we divided all performance tasks into three levels of complexity: easy (e.g., data entry), medium (e.g., calculations), called performance/medium, and difficult (e.g., formatting a diagram), called performance/difficult; these were done separately for the first test phase (near transfer performance) and the second test phase (far transfer performance). Both raters rated the level of complexity of the tasks with interrater agreement showing substantial agreement for Cohen's Kappas (Landis \& Koch, 1977; Kappa $=.68, S D=.12$ for tasks in the first and Kappa $=.89, S D=.08$ for tasks in the second performance test). We calculated performance sum scores for every participant and for each test phase, separately for easy, medium, and difficult tasks. (Because different numbers of subtasks appear for the different levels of difficulty, easy [not reported], medium, and difficult ratings cannot be directly compared. Thus, it is not possible to compare the absolute scores across the different levels of difficulty.) All analyses are based on these sum scores. Because we had developed hypotheses only for performance measures of medium and high difficulty, we did not include the easy tasks in further analyses. As discussed in the introduction, both theory and empirical research suggest that there should not be any significant differences between the training groups on easy tasks. This was, indeed, the case, as shown by analyses not reported because of space constraints.

Computer experience. We used three variables as control variables: The number of years of use of computers (years computer usage), the number of different programs the participants had worked with (number of programs), and whether they owned a computer or not (computer ownership). Because these three variables are not part of one dimension (ownership does not imply that one has worked many years with the computer and the number of years worked with a computer is not necessarily related to the fact that one knows different programs) - and we did not expect them to be highly correlated-we used them as three separate controls.

\section{Results}

\section{Preliminary Analyses}

Table 1 displays means, standard deviations, and intercorrelations of the study variables. The intercorrelations show substantial cross-time consistency in the performance variables. Not surprisingly, most of the computer experience variables were significantly related to the performance variables. Given these significant correlations, we used the computer experience variables as covariates in further analyses. Further, 


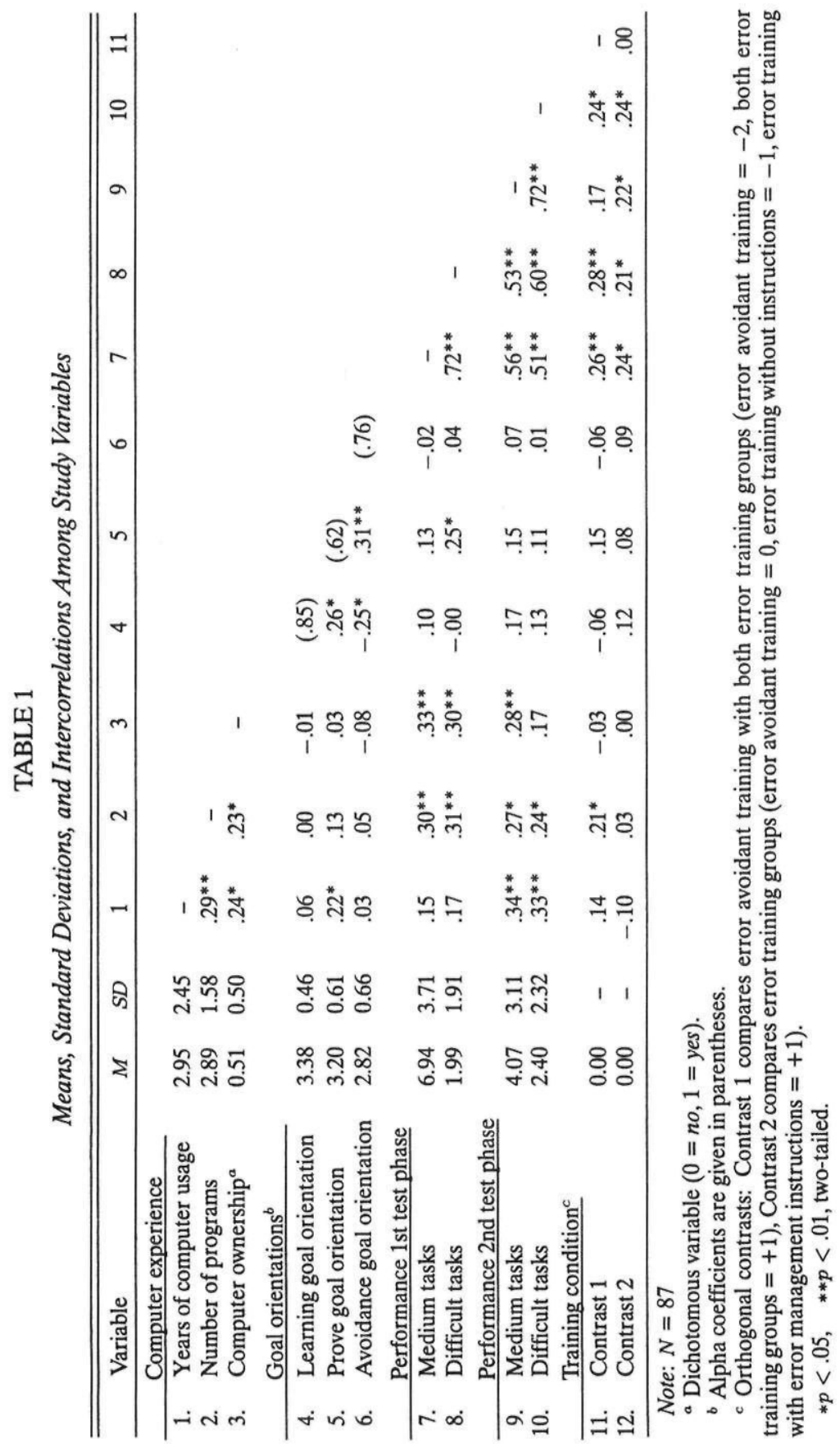


there were no direct relationships between goal orientation and performance: Out of 12 correlations between the goal orientation and the performance variables, only 1 was significant.

Because the participants were randomly distributed to the experimental groups, we did not expect any differences in computer experience and goal orientation. Indeed, there were no significant differences between training conditions in computer experience (data not shown, $F(2,84)=1.25, p=.29$ for years usage; $F(2,84)=2.01, p=.14$ for number of programs; $F(2,84)=0.04, p=.96$ for computer ownership). There were also no significant differences in the goal orientation variables (data not shown, $F(2,84)=0.76, p=.47$ for learning goal orientation; $F(2,84)=1.25, p=.29$ for prove goal orientation; $F(2,84)=0.44$, $p=.65$ for avoidance goal orientation).

\section{Performance Effects}

Hypothesis 1 predicted that error training with error management instructions leads to higher performance than the two other trainings. An overall multivariate analysis of covariance (MANCOVA) with the factor training (three groups) and the four performance variables as dependent variables (performance/medium and difficult for both phases [near and far transfer]) was conducted; we used years of computer usage, number of programs, and owning a computer as covariates. The MANCOVA showed an overall significant effect for the training groups (Wilks Lambda $F(8,156)=2.089, p=.04$ ). Planned contrasts (comparing the error training with error management instructions with the other two training conditions) revealed that the significant MANCOVA effect was due to error training with error management instructions producing better performance than (each of) the other two training groups; the latter two groups did not differ significantly. The results support our Hypothesis 1.

In the next step, we computed univariate analyses of covariance for the five performance variables separately controlling for computer experiences variables (cf. Figure 1 for the mean differences). We again included planned contrasts comparing error training with error management instructions with the other two training conditions. The effects were again in line with Hypothesis 1 (Table 2): We found significant performance differences between the training groups, in the first test phase as well as in the second test phase. Planned contrasts showed participants of the error training with error management instructions to perform significantly better compared to the other two groups; the latter two groups (error training without error management instructions and error avoidant training) did not differ from each other; this was true for 


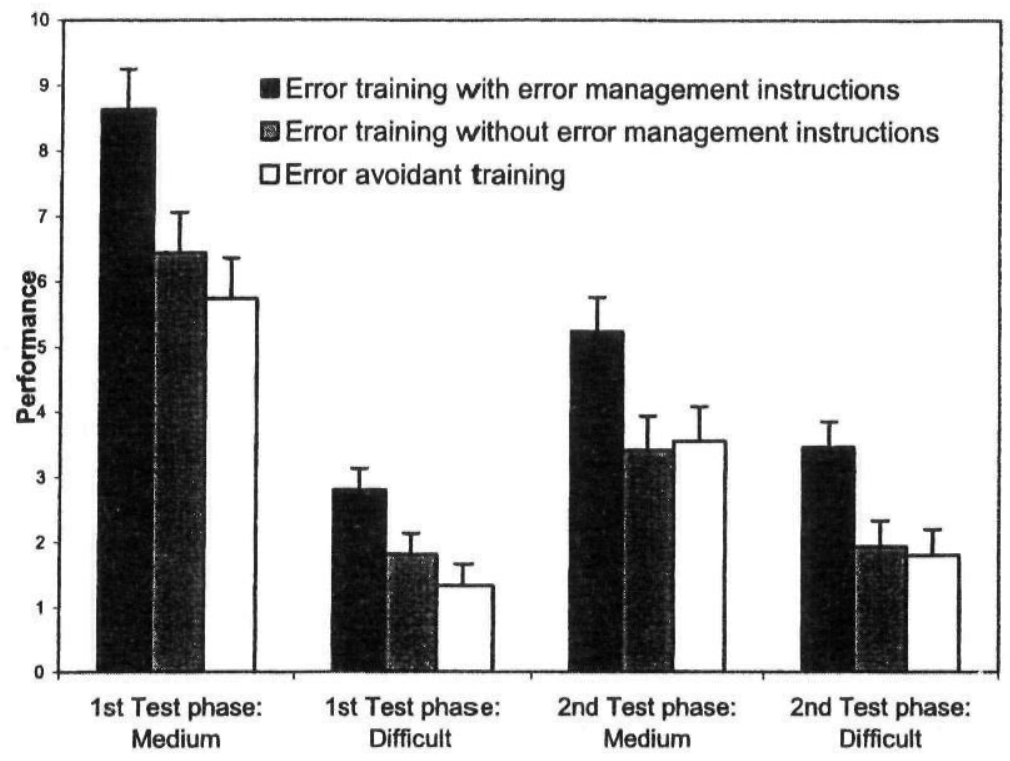

Figure 1: Means and Standard Errors of Performance Variables in Three Training Conditions Adjusted for Computer Experience Variables

\section{TABLE 2}

Observed Means and Standard Deviations of Performance Variables in the Three Training Conditions and Results of Univariate ANCOVAs

\begin{tabular}{|c|c|c|c|c|c|}
\hline & \multicolumn{2}{|c|}{ Error training } & \multirow{3}{*}{$\begin{array}{c}\text { Error } \\
\text { avoidant } \\
\text { training } \\
(n=29) \\
M(S D)\end{array}$} & \multirow[b]{3}{*}{$F(d f)$} & \multirow[b]{3}{*}{$\eta^{2}$} \\
\hline & $\begin{array}{c}\text { with error } \\
\text { management } \\
\text { instructions } \\
(n=29)\end{array}$ & $\begin{array}{c}\text { without error } \\
\text { management } \\
\text { instructions } \\
(n=29)\end{array}$ & & & \\
\hline & $M(S D)$ & $M(S D)$ & & & \\
\hline \multicolumn{6}{|l|}{ ANCOVAs 1st test phase: } \\
\hline Performance/medium & $8.72^{a}(3.58)$ & $6.52^{b}(3.56)$ & $5.59^{b}(3.38)$ & $6.01^{* *}(2,81)$ & .13 \\
\hline Performance/difficult & $2.85^{a}(2.07)$ & $1.86^{b}(1.81)$ & $1.24^{b}(1.53)$ & $5.43^{* *}(2,81)$ & .12 \\
\hline \multicolumn{6}{|l|}{ ANCOVAs 2nd test phase: } \\
\hline Performance/medium & $5.28^{a}(3.66)$ & $3.62^{b}(2.72)$ & $3.31^{b}(2.55)$ & $3.81^{*}(2,81)$ & .09 \\
\hline Performance/difficult & $3.48^{a}(3.01)$ & $2.10^{b}(1.52)$ & $1.62^{b}(1.80)$ & $5.51^{* *}(2,81)$ & .12 \\
\hline
\end{tabular}

Note: Means in the same row with different subscripts differ at $p<.05$; covariates: years of computer usage, number of programs, and computer ownership.

$* p<.05 \quad * * p<.01$. 
all four performance variables (Figure 1). The $\eta^{2}$ values were medium to high according to Cohen (1988). Cohen's $d$ values ranged from 0.63 (for second phase tasks of medium difficulty) to 0.90 (for first phase tasks of medium difficulty), comparing error training with error management instructions with error avoidant training.

Thus, we found substantial support for our hypothesis: Error training with error management instructions was the most successful training method in this experiment with sizeable effect sizes. The error management instructions were essential for the effectiveness of error training. Further, the positive performance effects of the error training with error management instructions on medium difficult and difficult tasks were stable over time.

\section{Interactions of Goal Orientation and Training Conditions}

Hypothesis 2 predicted an interaction effect of participants' goal orientations with training conditions on performance. More specifically, we expected learning goal orientation to be beneficial in the error training conditions and both prove and avoidance goal orientations to be beneficial in the error avoidant training. We tested this hypothesis using moderated regression analyses following Aiken and West (1991) and contrast coding as suggested by Cohen and Cohen (1983). In the first step, we entered computer experience variables as controls. In the second step, we modeled orthogonal contrasts (Contrast 1: error avoidant versus error training conditions; Contrast 2: error training with error management instructions versus error training condition without these instructions). In the third step, we entered goal orientation variables (centered around zero; Aiken \& West, 1991). In the final step, we entered the interaction term of interest (Contrast $1 \times$ goal orientation). The significance of interaction effects was evaluated using the increment in $R^{2}$ (Cohen $\&$ Cohen, 1983). We ran analyses separately for learning, prove, and avoidance goal orientations. To reduce the number of necessary analyses, we reduced the number of variables by aggregating the four dependant variables into a performance variable first phase and a performance variable second phase (summing $z$-scores of performance/medium, performance/difficult).

We conducted six moderated regression analyses and found three significant moderator effects (Table 3). In contrast to Hypothesis 2, there was no significant effect for learning goal orientation. However, there were two significant interaction effects for prove goal orientation, explaining $6 \%$ and $4 \%$ additional variance of first and second test phase performance, respectively. Further, there was one significant interaction effect for avoidance goal orientation ( $5 \%$ added explained variance 


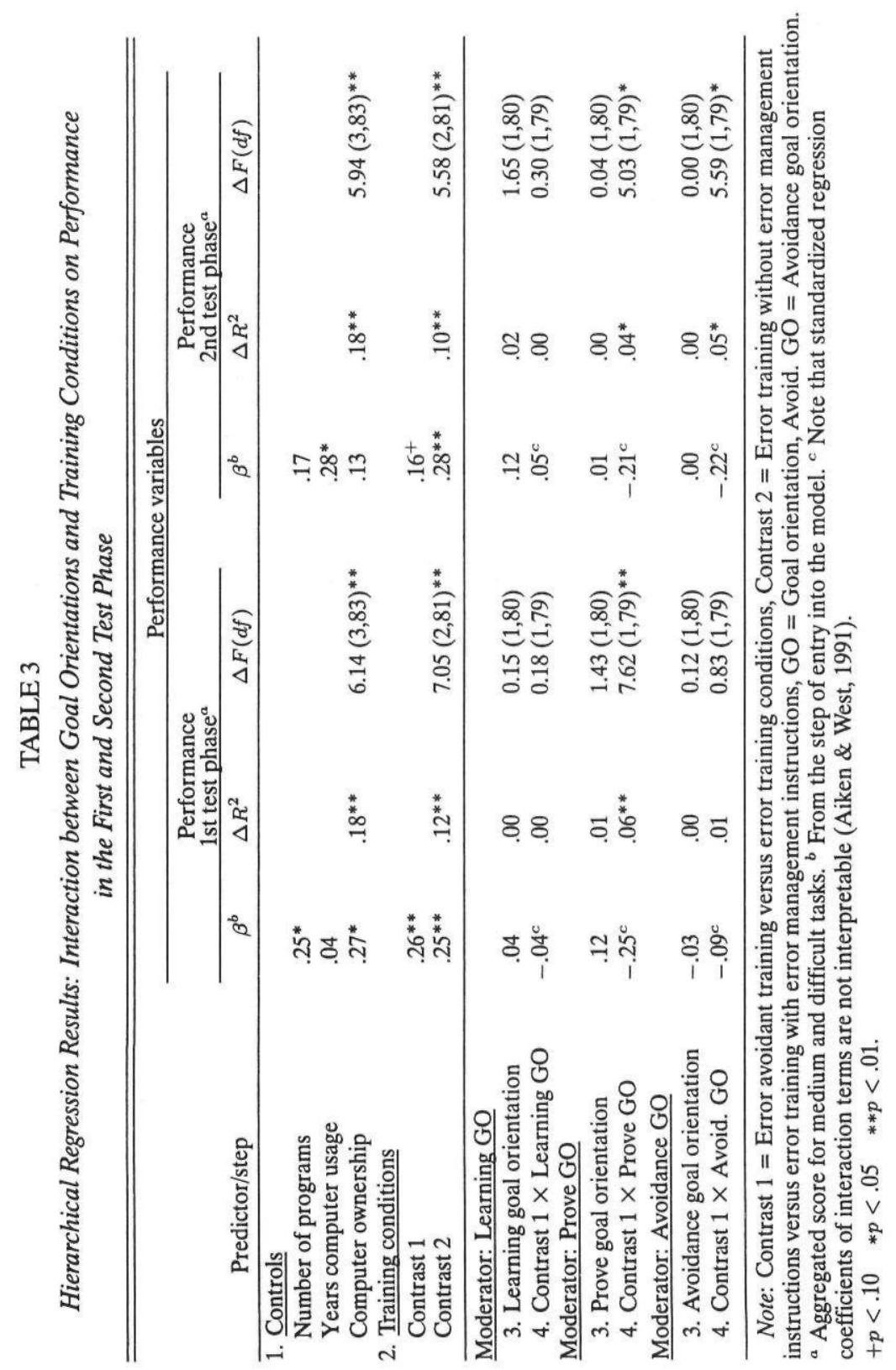


of second phase test performance). Thus, there was partial support for Hypothesis 2. The three significant interactions of goal orientation and training conditions are plotted in Figure 2 (based on Aiken \& West, 1991). They all show the same type of relationship. In each case, the highest performance scores appeared for the error training with error management instructions. Moreover, there were no differences between high and low prove (or avoidance) goal orientation in the two error training groups. The major differences appeared in the error avoidant condition: In each case, we hypothesized high prove (or avoidance) goal orientation to lead to better performance in this condition and, indeed, this was the case: The participants with high prove (and avoidance) goal orientation in this condition showed higher performance than those participants with low prove (and avoidance) goal orientation. Thus, Hypothesis 2 was partially supported. There was no support for our hypothesis on learning goal orientation (there were no significant moderator effects). There was support for Hypothesis 2 for prove goal orientation (two out of two potential interaction effects were significant). Support for avoidance goal orientation was somewhat weaker (one of the two potential interaction effects was significant); as expected, high avoidance goal orientation was beneficial and low avoidance goal orientation was detrimental to second phase performance in the error avoidant training.

\section{Discussion}

The function of errors in the learning process has been underemphasized in training research (Ivancic \& Hesketh, 1995). Error training is a new approach to use errors productively in the training process. This study tested the effectiveness of error training. We differentiated between an error training approach that included error management instructions (statements that emphasized the positive function of errors), error training without these instructions, and an error avoidant training that prevented trainees from making errors by providing detailed instructions of how to solve the tasks. The results indicated that a training design providing opportunities from making errors coupled with explicit instructions to encourage to learn from these errors has positive performance consequences in comparison to a training approach that does not allow people to make errors in the learning process. The performance superiority of the error training with error management instructions appeared in near transfer tests directly after the training, and in far transfer tests (one week after the training). Comparing the error training with error management instructions with the error avoidant training produced effect sizes from 0.63 to 0.91 , effect sizes that are surprisingly high, given 

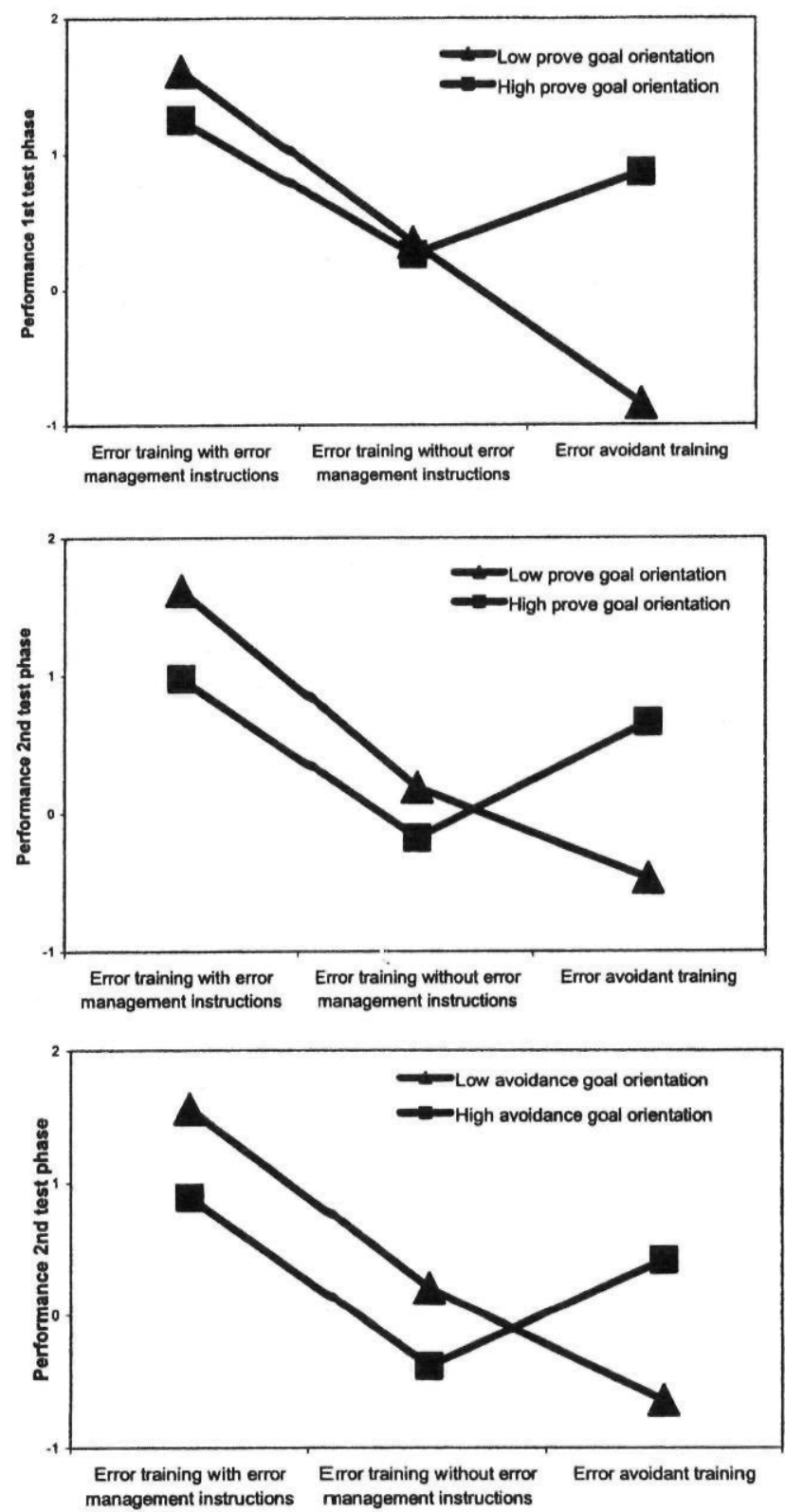

Figure 2: Significant Interaction Effects of Prove Goal Orientation/Avoidance Goal Orientation and Training Conditions on Performance in the First Phase (Near Transfer) and in the Second Phase (Far Transfer Performance); all scores aggregated for medium and difficult tasks after $z$-standardization 
that the comparison group was an alternative training and not a nontraining control group.

The positive results of error training are in line with earlier findings (Dormann \& Frese, 1994; Frese et al., 1991; Irmer, Pfeffer \& Frese, 1991; Ivancic \& Hesketh, 2000; Nordstrom et al., 1998; Wood, Kakebeeke, Debowski, \& Frese, 2000). In contrast to earlier studies on error training, however, the present study is more definitive: The study included a second test phase that measured transfer performance. Participants in the error training with error management instructions were higher on performance one week after the training. We also tested the role of error management instructions explicitly. The error training without instructions group showed significantly worse performance than the error training group with these instructions and was not significantly better than the error avoidant training. This speaks for our hypothesis that the error management instructions have an additional positive effect on learning from errors and that error training itself does not have these positive effects. We assume that the negative feedback function of errors has a positive function for developing a good mental model that can be used to regulate one's actions, only if the stressfulness of errors is reduced by the error management instructions.

We also examined attributes-treatment interactions and tested participants' goal orientation to moderate the effects of training conditions (Hypothesis 2). There was partial support for the moderator function of avoidance and prove goal orientation. Figure 2 presents the significant results. This partial support suggests that there is evidence for our hypothesis for prove and avoidance goal orientation. People with high prove and avoidance goal orientation showed better performance effects in the error avoidant training situation in which it was easy to "shine" (prove goal orientation) and in which one did not have to be anxious to make errors and fail (avoidance goal orientation) than people with low prove and avoidance goal orientation.

There was no support for the potential moderator learning goal orientation. Our hypothesis with regard to learning goal orientation was that high learning goal orientation should be beneficial for performance (and low learning goal orientation should be detrimental) in the error training conditions. The results suggest that error training is powerful enough to wipe out the effects of a personality variable. A possible interpretation of our results rests on the notion of strong versus weak situations. Mischel (1968) differentiated between strong and weak situations and argued that personality produces smaller effects in strong situations than in weak situations. Error training (particularly the condition with error management instructions) is probably the strongest situation because there are obvious difficulties to deal with tasks and the 
instructions present an explicit model on how a training should be done (through the instructions). Therefore, it is understandable that personality played a minor role in the error training groups. This also explains why there were no differences in prove and avoidance goal orientation in this training condition (cf. Figure 2).

\section{Implications for Theory}

The main contribution of this study underlines the crucial role of error management instructions for the effectiveness of error training. This has implications for (a) expertise, (b) self-regulation theories, and (c) error management. Comparisons between experts and novices show that experts possess a deeper and more abstract understanding of their knowledge domain and rely more on metacognitive knowledge (Eteläpelto, 1993; Hinds, Patterson, \& Pfeffer, 2001; McKeithen, Reitman, Rueter, \& Hirtle, 1981). We suggest that error training helps in developing such a deeper and more abstract understanding. Error training with error management instructions provides metacognitive tools that can be useful in future task accomplishment processes. Thus, basic principles of error training enable participants to approach tasks in a way similar to an expert's approach. Moreover, Ericsson and Charness (1994) argued that deliberate practice is essential for developing expertise. Deliberate practice is a regular, effortful activity explicitly aiming at improving one's skills and focusing on those parts of the task that are the most difficult to do. We think that error training is related to deliberate practice in two ways. First, those areas of task completion that produce the most errors are usually also the most difficult to do. Thus, deliberate practice is most likely the practice of error prone task areas. Second, the depth of practice is higher with error training; however, it needs to be complemented by error management instructions to reduce the negative emotional and motivational effects of making errors, as shown by this experiment. We propose that deliberate practice could also make use of such error management instructions.

Self-regulation theories, such as the Feedback Intervention Theory by Kluger and DeNisi (1996), have suggested that performance is enhanced if feedback encourages trainees to be task oriented and that performance is decreased if feedback leads people to think about themselves. Error management instructions may help keep trainees' attention on the task and away from the self. Without error management instructions, people have a tendency to think about their selves when they make an error because errors threaten their self-confidence. In a similar way, error management instructions might help trainees to increase their self-regulatory capacity (in the sense of resource allocation by Kanfer \& 
Ackerman, 1989), which then leads to more on-task attention and unrestricted use of cognitive resources. Similarly, Sonnentag (1998) found that expert performance is characterized by a lack of task-irrelevant cognitions. The error management instructions might help trainees to adopt a task-oriented working style and prevent them from ruminating about negative thoughts and frustration due to errors. Additional issues that need to be studied in future studies are an increase of self-efficacy as a result of error training with error management instructions and possible interactions with cognitive ability.

The error management concept is an alternative to error prevention (Frese \& Zapf, 1994); in error prevention, the erroneous action is prevented, in error management, the negative consequences of the error are reduced. The negative consequences may be contained (Helmreich, Merritt \& Wilhelm, 1999, called this trapping the error) and the error consequences can be mitigated. Error management instructions help to manage errors because they may decrease the additional task of handling one's emotional reaction to errors. Modern stress theory suggests that one facet of stress is that information processing resources allocated to the task are reduced when additional tasks, such as negative emotions, worrying, ruminating, and so forth have to be dealt with (Hockey, 1996; Meijman \& Mulder, 1998). In this way, error management instructions reduce the worrying and ruminating about errors and, thereby, make it possible to deal with both tasks and errors better. When learning to deal with errors is a part of the training process, dealing with the inevitable occurrence of errors in real life tasks is integrated into the training of a new software system. Therefore, transfer should be enhanced. In general, we think that future error training research should examine these various processes in more detail to understand better why error training shows positive performance effects.

\section{Strengths and Limitations of the Study}

Baldwin and Ford (1988) have called for studies that examine the maintenance and generalizability of skills acquired during the training program in the job setting. Our transfer results represent promising evidence that error management instructions help to maintain and generalize skills.

Our study, as most other training studies, used a student sample; this might have influenced the results. Because students are supposed to focus on learning, there might have been a ceiling effect and restricted variance in learning goal orientation. In addition, asking for volunteers to participate in a study on training might have oversampled those students who are interested in learning new skills. 
Prove goal orientation showed a low reliability, which was clearly below the usual .70 level. Should other studies replicate this lower than expected alpha level, it would be useful to develop a more reliable measure of prove orientation.

\section{Implications for Practice and Future Research}

Error training with error management instructions is an effective training procedure. One practical implication of this study is to take a more positive view of errors in instructional design. Although errors are usually frustrating to participants in training, this should not necessarily lead to the view that errors should be minimized. Errors can have a positive function for learning to perform and transfer when people are taught to use errors as a chance to learn and when they are taught to reduce the negative function of errors, as was done in the error training group with error management instructions.

This does not mean that we suggest to use blind trial and error as a strategy to learn. As a matter of fact, unsystematic trial and error without any explicit hypothesis and without an idea of what one wants to test, may actually have a negative effect on learning (van der Linden, Sonnentag, Frese, \& van Dyck, 2001). Another limiting condition may be severe memory impairment. As a matter of fact, there is evidence that with memory impaired participants, it may be better to provide errorless learning (Wilson, Baddeley, \& Evans, 1994). The same may also apply for training participants with low mental ability (Gully et al., 2002).

Our results suggest, however, that practitioners may well take notice of the positive function of errors in the training process of normal adults and use elements of error training within their training designs. This does not necessarily imply that the full training should be modeled along the lines of error training suggested in this article. It may well be possible that error management training should be included in a later phase of the learning process. In the very beginning of training, a guided approach may be useful so that participants learn the basics; then an error training with error management instructions module should be added. Wood et al.'s (2000) study suggests such an approach. Wood et al. (2000) taught an electronic search task with a guided mastery training in the beginning phase. Adding error training with error management instructions in a second phase (they called it enactive exploration) led to higher performance than when error training was not given. Thus, it is in principle possible to combine the error training approach with more traditional approaches. Irmer et al. (1991) included a 1-day module of error training with error management instruction in a traditional thre3day training course of a software training institute; they found that this 
led to higher performance than the 3-day training course without the error training module; in both cases, the traditional part of the training course was given by the same highly experienced teacher of this training institute.

The most important reason why error training with error management instructions should be used in practice is because errors appear whenever one transfers knowledge acquired in training to the normal work situation (Hesketh, 1997). Our study has shown far transfer after one week to be positively affected by error training with error management instructions. We propose that it is useful to go down this path somewhat further and investigate the usefulness of error training on transfer in the normal work situation of employees. We assume that error training with error management instructions has a positive impact on transfer of knowledge to the work place as well and that this effect may be even stronger than the direct performance effect after training.

Aspects of error training have begun to appear in crew resource management training in aviation (Helmreich et al., 1999). Learning from errors has been a catchword in the attempt to deal with errors in the medical setting (Kohn, Corrigan, \& Donaldson, 2000). We hope that our work on error training can be used in these setting to develop more concrete and better models of how learning from errors occurs and can be supported in simulation training.

On a more speculative note, we would also like to argue that the error training approach is practically useful because it opens people up for change. One of the functions of errors is to produce conscious processing even of prior habitual responses (Lord \& Levy, 1994). One of the factors that keeps people from being open to change at work is that new approaches are effortful and increase the chances to make errors (after all, old habits are kept because they do not require one to think about them; Bargh \& Chartrand, 1999; Kahneman, 1973). Possibly, one of the functions of error training with error management instructions is to open people up to new ideas and to make them explore the system when an error occurs (Dormann \& Frese, 1994). Moreover, we assume that people who have been trained with an error training approach may generalize and may have a "better" attitude towards learning from errors.

There are limiting factors for an error training approach. The most important one is that error training only works if the training environment provides feedback. This is the case in learning computer software skills because most modern software includes meaningful feedback so that people can actually learn from their errors. This is also the case for learning to drive in a simulator (Ivancic \& Hesketh, 2000). Ivancic and Hesketh (2000) have explored the function of making errors versus being taught how to deal with them (they call the latter group "guided 
error training"). Their study suggests that learning is better when people are allowed to make the errors themselves and that guiding people through other people's errors does not have the same positive effect as error training. Another limiting factor shown to be important in this study is the function of error management instructions. It is plausible that instructions have the function to reduce the threat and stressfulness of making errors. We propose that the error training approach has a positive function only in an environment that is safe to explore and in which people do not have to worry about negative consequences of making errors (Sitkin, 1992).

Although our study has shown error management instructions to have a positive function for performance, further research is needed to determine the reasons for this. Future studies should investigate in which way the instructions work, how managing errors can assure the performance effects of error training, and in which areas of skill development error training can be used (e.g., social skills training).

\section{REFERENCES}

Aiken LS, West SG. (1991). Multiple regression: Testing and interpreting interactions. Newbury Park, CA: Sage.

Ames C, Archer J. (1988). Achievement goals in the classroom: Student's learning strategies and motivation. Journal of Educational Psychology, 80, 260-267.

Ausubel DP. (1968). Educational psychology: A cognitive view. New York: Holt, Rinehart and Winston.

Baldwin TT, Ford JK. (1988). Transfer of training: A review and directions for future research. PERSONNEL PSYCHOLOGY, 41, 63-105.

Bargh JA, Chartrand TL. (1999). The unbearable automaticity of being. American Psychologist, 54, 462-479.

Boyle KA, Klimoski RJ. (1995). Toward an understanding of goal orientation in a training context. Paper presented at the 10th Annual Conference of the Society for Industrial and Organizational Psychology, Orlando, FL.

Brett JF, VandeWalle D. (1999). Goal orientation and goal content as predictors of performance in a training program. Journal of Applied Psychology, 84, 863-973.

Brodbeck FC, Zapf D, Prümper J, Frese M. (1993). Error handling in office work with computers: A field study. Journal of Occupational and Organizational Psychology, 66, 303-317.

Brown KG, Kozlowski SWJ. (1997). Self-evaluation and training outcomes: Training strategies and goal orientation effects. 12th Annual Conference of the Society for Industrial and Organizational Psychology, St. Louis, MO.

Bruner JS. (1966). Toward a theory of instruction. Cambridge, MA: Harvard University Press.

Cannon-Bowers JA, Rhodenizer L, Salas E, Bowers CA. (1998). A framework for understanding prepractice conditions and their impact on learning. PERSONNEL PSYCHOLOGY, 51, 291-320.

Carroll, JM, Mack RL, Lewis CH, Grischkowsky NL, Robertson SR. (1985). Exploring a word processor. Human-Computer Interaction, 1, 283-307. 
Carroll JM, Smith-Kerker PL, Ford JR, Mazur-Rimetz SA. (1987). The minimal manual. Human-Computer Interaction, 3, 123-153.

Cohen J. (1988). Statistical power analysis for the behavioral sciences. New York: Erlbaum.

Cohen J, Cohen P. (1983). Applied multiple regression/correlation analysis for the behavioral sciences. Hillsdale, NJ: Erlbaum.

Colquitt JA, Simmering MJ. (1998). Conscientiousness, goal orientation, and motivation to learn during the learning process: A longitudinal study. Journal of Applied Psychology, 83(4), 654-665.

Craig FIM, Lockhart RS. (1972). Levels of processing: A framework for memory research. Journal of Verbal Learning and Verbal Behavior, 11, 671-684.

Debowski S, Wood RE, Bandura A. (2001). Impact of guided exploration and enactive exploration on self-regulatory mechanisms and information acquisition through electronic search. Journal of Applied Psychology, 86, 1129-1141.

Dormann T, Frese M. (1994). Error training: Replication and the function of exploratory behavior. International Journal of Human-Computer Interaction, 6(4), 365-372.

Dweck CS. (1986). Motivational processes affecting learning. American Psychologist, 41, 1040-1048.

Dweck CS. (1989). Motivation. In Lesgold A, Glaser R (Eds.), Foundations for a psychology of education. Hillsdale, NJ: Erlbaum.

Dweck CS, Leggett EL. (1988). A social-cognitive approach to motivation and personality. Psychological Review, 95, 256-273.

Elliot AJ, Harackiewicz JM. (1996). Approach and avoidance achievement goals and intrinsic motivation: A mediational analysis. Journal of Personality and Social Psychology, 70(3), 461-475.

Ellis A. (1962). Reason and emotion in psycholotherapy. New York: Lyle Stuart.

Ericsson KA, Charness N. (1994). Expert performance: Its structure and acquisition. American Psychologist, 49, 725-747.

Ericsson KA, Krampe RT, Tesch-Römer C. (1993). The role of deliberate practice in the acquisition of expert performance. Psychological Review, 100, 363-406.

Eteläpelto A. (1993). Metacognition and the expertise of computer program comprehension. Scandinavian Journal of Educational Research, 37, 243-254.

Eysenck HJ. (1996). Personality and the experimental study of education. European Journal of Personality, 10, 427-439.

Farr JL, Hofmann DA, Ringenbach KL. (1993). Goal orientation and action control theory: Implications for industrial and organizational psychology. International Review of Industrial and Organizational Psychology, 8, 191-232.

Ford JK, Smith EM, Weissbein DA, Gully SM, Salas E. (1998). Relationships of goal orientation, meta-cognitive activity, and practice strategies with learning ourcomes and transfer. Journal of Applied Psychology, 83, 218-233.

Frese M. (1995). Error management in training: Conceptual and empirical results. In Zucchermaglio C, Bagnara S, Stucky SU (Eds.), Organizational learning and technological change (pp. 112-124). Berlin: Springer.

Frese M, Altmann A. (1989). The treatment of errors in learning and training. In Bainbridge L, Ruiz Quintanilla A (Eds.), Developing skills with information technology (pp. 65-86). Chichester, U.K.: Wiley.

Frese M, Brodbeck FC, Heinbokel T, Mooser C, Schleiffenbaum E, Thiemann P. (1991). Errors in training computer skills: On the positive function of errors. Human Computer Interaction, 6, 77-93.

Frese M, Zapf D. (1994). Action as the core of work psychology: A German approach. In Triandis HC, Dunnette MD, Hough L (Eds.), Handbook of industrial and organizational psychology (Vol. 4, pp. 271-340). Palo Alto, CA: Consulting Psychologists Press. 
Gick ML, Holyoak KJ. (1987). The cognitive basis of knowledge transfer. In Cormier SM, Hagman JD (Eds.), Transfer of learning: Contemporary research and applications (pp. 9-46). San Diego, CA: Academic Press.

Gick ML, McGarry SJ. (1992). Learning from mistakes: Inducing analogous solution failures to a source problem produces later successes in analogical transfer. Journal of Experimental Psychology: Learning, Memory, and Cognition, 18(3), 623-639.

Goldstein IL. (1992). Training in work organizations. In Dunette MD, Hough LM (Eds.), Handbook of industrial and organizational psychology, 2nd ed. (Vol. 2, pp. 507-619). Palo Alto, CA: Consulting Psychologists Press.

Greif S, Janikowski A. (1987). Aktives Lernen durch systematische Fehlerexploration oder programmiertes Lernen durch Tutorials? [Active learning through systematic error exploration or programmed learning through tutorials?]. Zeitschrift für Arbeits- und Organistionspsychologie, 31, 94-99.

Greif S, Keller H. (1990). Innovation and the design of work and learning environments: The concept of exploration in human-computer interaction. In West MA, Farr JL (Eds.), Innovation and creativity at work (pp. 231-249). New York: Wiley.

Gully SM, Payne SC, Koles KLK, Whiteman JAK. (2002). The impact of error training and individual differences on training outcomes: An attribute-treatment interaction perspective. Journal of Applied Psychology, 87, 143-155.

Heimbeck D, Frese M, Sonnentag S, Van Dyck C, Van der Linden D. (2003). Goal orientation: A comparison between two instruments and their relationship with achievement motivation. University of Amsterdam: Submitted for publication.

Helmreich RL, Merritt AC, Wilhelm JA. (1999). The evolution of crew resource management in commercial aviation. International Journal of Aviation Psychology, 9, 19-32.

Hesketh B. (1997). Dilemmas in training for transfer and retention. Applied Psychology: An International Review, 46, 317-339.

Hinds PJ, Patterson M, Pfeffer J. (2001). Bothered by abstraction: The effect of expertise on knowledge transfer and subsequent novice performance. Journal of Applied Psychology, 86, 1232-1243.

Hockey R. (1996). Skilled performance and mental workload. In Warr P (Ed.), Psychology at work (4th ed., pp. 13-39). London: Penguin.

Irmer C, Pfeffer S, Frese M. (1991). Konsequenzen von Fehleranalysen für das Training: Das Fehlertraining [Consequences of error analyses for training: The error training]. In Frese M, Zapf D (Eds.), Fehler bei der Arbeit mit dem Computer (pp. 151165). Bern, Switzerland: Huber.

Ivancic K, Hesketh B. (1995). Making the best of errors in training. Training Research Journal, 1, 103-125.

Ivancic K, Hesketh B. (2000). Learning from errors in a driving simulation: Effects on driving skill and self-confidence. Ergonomics, 43, 1966-1984.

Kahneman D. (1973). Attention and effort. Englewood Cliffs, New Jersey: Prentice Hall.

Kanfer R, Ackerman PL. (1989). Motivation and cognitive abilities: An integrative/aptitudetreatment interaction approach to skill acquisition. Journal of Applied Psychology, 74, 657-690.

Kluger AN, DeNisi A. (1996). The effects of feedback interventions on performance: A historical review, a meta-analysis, and a preliminary feedback intervention theory. Psychological Bulletin, 119, 254-284.

Kohn LT, Corrigan JM, Donaldson MS (Eds.). (2000). To err is human. Washington, D.C.: National Academy Press.

Kraiger K. (1995). Integrating training research. Training Research Journal, 1, 5-16.

Landis JR, Koch GG. (1977). The measurement of observer agreement for categorial data. Biometrics, 33, 150-174. 
Lazonder AW, Van der Meij H. (1993). The minimal manual: Is less really more? International Journal of Man Machine Studies, 39(5), 629-752.

Lazonder AW, Van der Meij H. (1994). Effect of error information in tutorial documentation. Interacting with Computers, 6(1), 23-40.

Lord RG, Levy PE. (1994). Moving from cognition to action: A control theory perspective. Applied Psychology: An International Review, 43, 335-366.

McKeithen KB, Reitman JS, Rueter HH, Hirtle SC. (1981). Knowledge organization and skill differences in computer programmers. Cognitive Psychology, 13, 307-325.

McClelland DC. (1987). Human motivation. Cambridge, UK: Cambridge University Press.

Meijman TF, Mulder G. (1998). Psychological aspects of workload. In Drenth PJD, Thierry H, De Wolff CJ (Eds.), Handbook of work and organizational psychology, 2nd ed. (Vol. 1, pp. 5-33). London: Psychology Press.

Mischel W. (1968). Personality and assessment. New York: Wiley.

Nordstrom CR, Wendland D, Williams KB. (1998). "To err is human": An examination of the effectiveness of error management training. Journal of Business and Psychology, $12,269-282$.

Sitkin SB. (1992). Learning through failure: The strategy of small losses. Research in Organizational Behavior, 14, 231-266.

Skinner BF. (1953). Science and human behavior. New York: The Free Press.

Skinner BF. (1968). The technology of teaching. New York: Meredith Corporation.

Snow RE. (1986). Individual differences and the design of educational programs. American Psychologist, 41, 1029-1039.

Sonnentag S. (1998). Expertise in professional software design: A process study. Journal of Applied Psychology, 83, 703-715.

Tannenbaum SI, Yukl G. (1992). Training and development in work organizations. Annual Review of Psychology, 43, 399-441.

Van der Linden D, Sonnentag S, Frese M, Van Dyck C. (2001). Exploration strategies, performance, and error consequences when learning a complex computer task. Behaviour and Information Technology, 20, 189-198.

VandeWalle D. (1997). Development and validation of a work domain goal orientation instrument. Educational and Psychological Measurement, 57(6), 995-1015.

Wilson BA, Baddeley A, Evans J. (1994). Errorless learning in the rehabilitation of memory impaired people. Neuropsychological Rehabilitation, 4, 307-326.

Wood RE, Kakebeeke BM, Debowski S, Frese M. (2000). The impact of enactive exploration on intrinsic motivation, strategy, and performance in electronic search. Applied Psychology: An International Review, 49, 263-283.

Zapf D, Brodbeck FC, Frese M, Peters H, Prümper J. (1992). Errors in working with computers: A first validation of a taxonomy for observed errors in a field setting. International Journal of Human-Computer Interaction, 4, 311-339. 
Copyright of Personnel Psychology is the property of Blackwell Publishing Limited and its content may not be copied or emailed to multiple sites or posted to a listserv without the copyright holder's express written permission. However, users may print, download, or email articles for individual use. 